\title{
Nanoscale Chemical Analysis of Individual Subcellular Structures
}

\author{
Daniel T. Chiu \\ Department of Chemistry, University of Washington, Seattle, WA 98195-1700.
}

\begin{abstract}
Although powerful imaging methods exist for studying the morphologies of cells and subcellular architectures, there exists no general strategy to probe the biochemical compositions of such an ultrasmall structure at the level of single copies. Figure 1A shows an example illustrating the power of electron microscopy (EM) for visualizing nanometer scale structures within cells. EM provides relatively static information, but the use of optical microscopy, such as scanning confocal and two-photon microscopy, has provided detailed information on cellular dynamics. Despite the power of microscopy in providing high-resolution visual images, it offers little chemical information about the subcelluar compartments that are being visualized. To understand the localization and function of complex cellular machineries, signaling pathways, and metabolic activities within the cell, we must have at our disposal a versatile and powerful set of analytical toolkits capable of providing comprehensive biochemical information about the nanometer-scale structures that we can visualize so well with microscopy.
\end{abstract}

The primary reason for this lack of techniques in extracting chemical information from nanoscale subcellular structures lies in the minute amount of samples available for analysis. A typical single organelle may range in diameter from tens of nanometers to a couple micrometers, with a corresponding volume of $\sim 6 \times 10^{-20} \mathrm{~L}$ (e.g for a 50 -nm synaptic vesicle) to $\sim 8 \times 10^{-15} \mathrm{~L}$ (e.g. for a $2-\mu \mathrm{m}$ mitochondrion). Within a volume of 6 $\times 10^{-20} \mathrm{~L}$, even at a high concentration of $100 \mathrm{mM}$, the number of molecules present is only $\sim 3600$. At this small length scales, most proteins would be present as a single copy or in a few copies. This extremely small amount of volume, limited number of molecules, and the complex mixtures of contained molecules within each subcellular compartment necessitate an approach that is both highly sensitive and capable of isolating each components of the mixture for quantitation and characterization. No technology exists currently for addressing this demanding challenge.

Recently, our group has embarked on the development of a comprehensive strategy for analyzing ultrasmall subcellular structures and compartments from single cells. This strategy relies on the ability to isolate nanometer-size structure from single cells using a combination of laser-based methods (see Figure 1B-1E), on the possibility to carry out chemical reactions in the nanoscale within sub-picoliter and sub-femtoliter volumes of solution, and on the capability to fabricate and integrate nanofluidic systems for performing complex analytical operations. We believe a strategy that offers comprehensive biochemical information on individual subcellular compartments with high spatial resolution will be a powerful complement to current bulk techniques for studying biological systems, which offer little spatial information with regard to the 
intracellular localization of proteins and metabolites. These bulk techniques are also blind to any variations that exist among these discrete biological samples. Methodologies that can provide a complete chemical inventory contained within nanometer-sized subcellular compartments with microscopic resolutions will be an invaluable tool for tackling the complex biological questions we face.
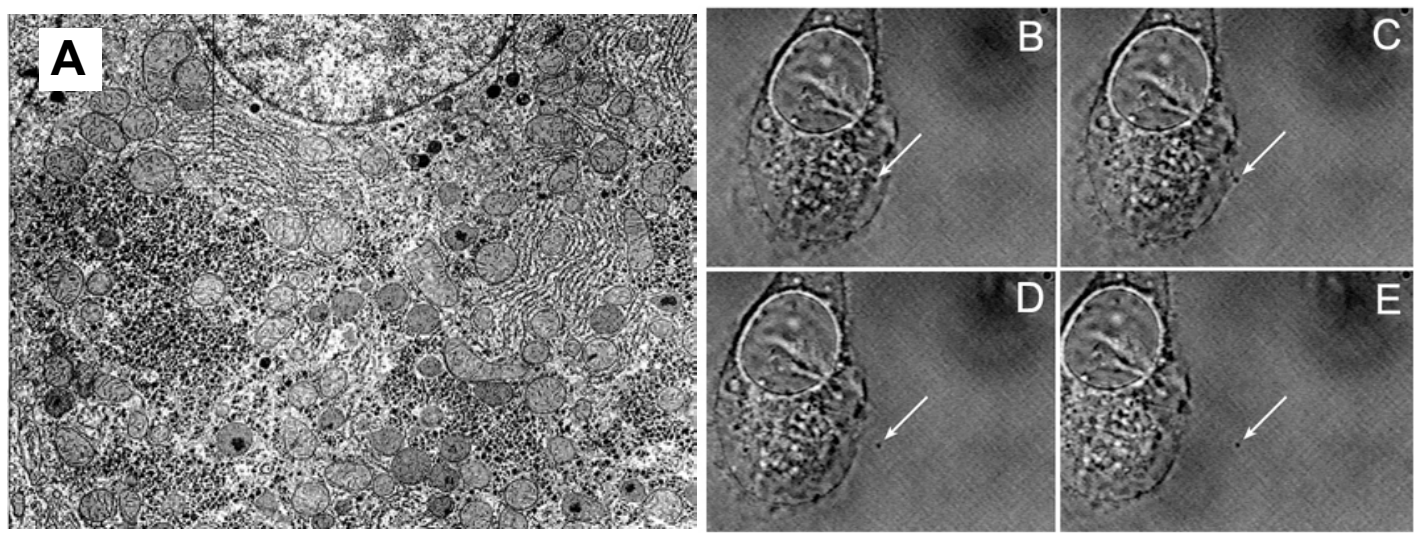

Figure 1. (A) Electron micrograph showing the interior of a cell. (B) - (E) is a video sequence showing the isolation of a single organelle (arrow) measuring one to two hundred nanometers from a $\mathrm{CHO}$ cell.

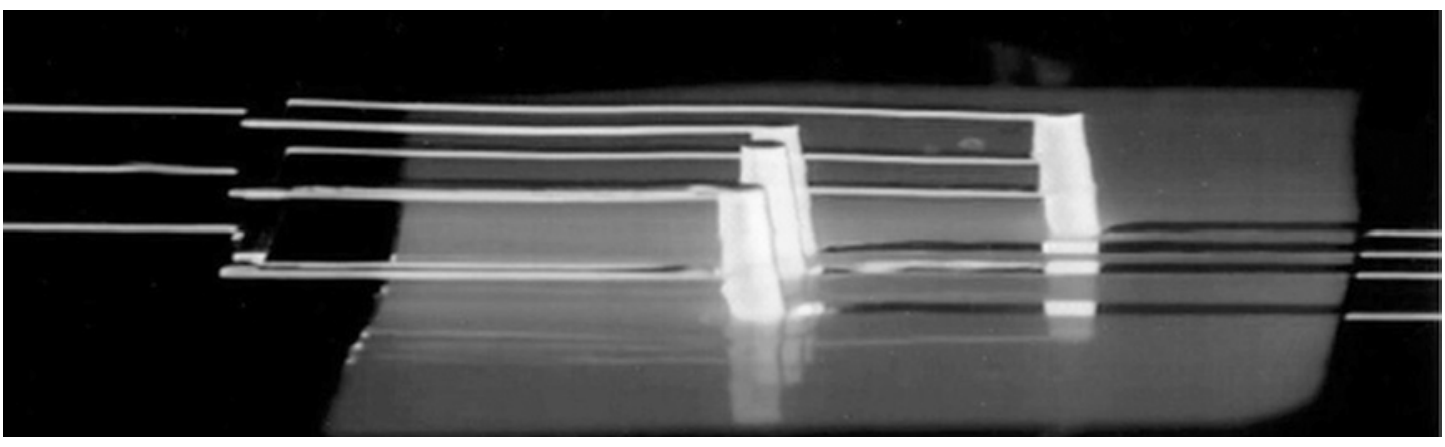

Figure 2. Example of a complex three-dimensional microfluidic system, in which the channels were filled with a fluorescent dye for visualization. The whitish membrane is a polycarbonate size filter. 